\title{
A new, three-dimensional geometric morphometric approach to assess egg shape
}

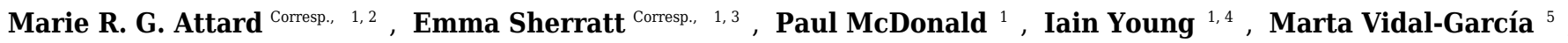 \\ Stephen Wroe ${ }^{1}$ \\ ${ }^{1}$ Zoology Department, School of Environmental and Rural Science, University of New England, Armidale, New South Wales, Australia \\ 2 Department of Animal and Plant Sciences, University of Sheffield, Sheffield, South Yorkshire, United Kingdom \\ 3 Department of Ecology and Evolutionary Biology, School of Biological Sciences, The University of Adelaide, Adelaide, South Australia, Australia \\ 4 School of Life and Environmental Sciences, Faculty of Science, University of Sydney, Sydney, New South Wales, Australia \\ 5 Ecology and Evolution, Research School of Biology, Australian National University, Canberra, ACT, Australia \\ Corresponding Authors: Marie R. G. Attard, Emma Sherratt \\ Email address: marie.r.g.attard@gmail.com, emma.sherratt@gmail.com
}

This paper proposes a new methodology to quantify patterns of egg shape variation using geometric morphometrics of three-dimensional landmarks captured on digitally reconstructed eggshells and demonstrates its performance in capturing shape variation at multiple biological levels. This methodology offers unique benefits to complement established linear measurement or two-dimensional (2D) contour profiling techniques by (i) providing a more precise representation of eggshell curvature by accounting for variation across the entire surface of the egg; (ii) avoids the occurrence of correlations from combining multiple egg shape features; (iii) avoids error stemming from projecting a highly-curved 3D object into 2D space; and (iv) enables integration into 3D workflows such as finite elements analysis. To demonstrate, we quantify patterns of egg shape variation and estimate morphological disparity at multiple biological levels, within and between clutches and among species of four passerine species of different lineages, using volumetric dataset obtained from micro computed tomography (micro-CT). The results indicate that species broadly have differently shaped eggs, but with extensive withinspecies variation so that all four-focal species occupy a range of shapes. Within-species variation is attributed to between-clutch differences in egg shape; within-clutch variation is surprisingly substantial. Recent comparative analyses that aim to explain shape variation among avian taxa have largely ignored potential biases due to within-species variation, or use methods limited to a narrow range of egg shapes. Through our approach, we suggest that there is appreciable variation in egg shape across clutches and that this variation needs to be accounted for in future research. The approach developed in this study to assess variation in shape is freely accessible and can be applied to any spherical-to-conical shaped object, including eggs of non-avian dinosaurs and reptiles through to other extant 
taxa such as poultry. 


\section{A new, three-dimensional geometric morphometric approach to assess egg}

\section{2 shape}

3 Marie R. G. Attard ${ }^{* 1,2}$, Emma Sherratt ${ }^{* 1,3}$, Paul McDonald ${ }^{1}$, Iain Young ${ }^{1,4}$, Marta Vidal-García ${ }^{5}$ 4 and Stephen Wroe ${ }^{1}$

$6 \quad{ }^{1}$ Zoology Department, School of Environmental and Rural Science, University of New England, 7 Armidale, NSW, 2351, Australia

$8 \quad{ }^{2}$ Department of Animal and Plant Sciences, University of Sheffield, Sheffield S10 2TN, UK

$9{ }^{3}$ Department of Ecology and Evolutionary Biology, School of Biological Sciences, The

10 University of Adelaide, Adelaide, South Australia 5005, Australia

$11{ }^{4}$ School of Life and Environmental Sciences, Faculty of Science, University of Sydney, NSW

12 2006, Australia

135 Ecology and Evolution, Research School of Biology, The Australian National University,

14 Canberra, ACT, 2601 Australia

$15{ }^{*}$ Co-first Author. These authors contributed equally to this work.

17 Corresponding Authors:

18 Marie Attard*1,2

19 Department of Animal and Plant Sciences, University of Sheffield, Sheffield S10 2TN, UK,

20 Email address: marie.r.g.attard@gmail.com

21 Emma Sherratt ${ }^{* 1,3}$

22 Department of Ecology and Evolutionary Biology, School of Biological Sciences, The 23 University of Adelaide, Adelaide, South Australia 5005, Australia 
24 Email address: emma.sherratt@gmail.com

25 Short title: Three-dimensional egg shape

26 Abstract

27

28 This paper proposes a new methodology to quantify patterns of egg shape variation using

29 geometric morphometrics of three-dimensional landmarks captured on digitally reconstructed 30 eggshells and demonstrates its performance in capturing shape variation at multiple biological

31 levels. This methodology offers unique benefits to complement established linear measurement 32 or two-dimensional (2D) contour profiling techniques by (i) providing a more precise 33 representation of eggshell curvature by accounting for variation across the entire surface of the 34 egg; (ii) avoids the occurrence of correlations from combining multiple egg shape features; (iii) 35 avoids error stemming from projecting a highly-curved 3D object into 2D space; and (iv) enables 36 integration into 3D workflows such as finite elements analysis. To demonstrate, we quantify 37 patterns of egg shape variation and estimate morphological disparity at multiple biological 38 levels, within and between clutches and among species of four passerine species of different 39 lineages, using volumetric dataset obtained from micro computed tomography (micro-CT). The results indicate that species broadly have differently shaped eggs, but with extensive within-

41 species variation so that all four-focal species occupy a range of shapes. Within-species variation 42 is attributed to between-clutch differences in egg shape; within-clutch variation is surprisingly 43 substantial. Recent comparative analyses that aim to explain shape variation among avian taxa

44 have largely ignored potential biases due to within-species variation, or use methods limited to a 45 narrow range of egg shapes. Through our approach, we suggest that there is appreciable variation 46 in egg shape across clutches and that this variation needs to be accounted for in future research. 
47 The approach developed in this study to assess variation in shape is freely accessible and can be

48 applied to any spherical-to-conical shaped object, including eggs of non-avian dinosaurs and

49 reptiles through to other extant taxa such as poultry.

\section{Introduction}

53 Avian egg shape diversity is a well-known biological phenomenon reflecting taxonomic

54 diversity (Olsen, Cunningham \& Donnelly, 1994; Stoddard et al., 2017), ranging from the nearly

55 spherical eggs of owls (Hoyt, 1976), slightly pointed eggs of domestic fowl (Havlíček et al.,

56 2008), to the extremely pointed eggs of certain waders, alcids and penguins (Birkhead et al.,

57 2017; Stoddard et al., 2017). Various hypotheses have been proposed to explain the evolution of 58 specific egg shapes (Rensch, 1947; Andersson, 1978; Bain \& Solomon, 1991; Attard et al., 2017;

59 Deeming, 2017; Stoddard et al., 2017), but so far only explain a small proportion of variation in 60 egg shape (Deeming, 2017; Stoddard et al., 2017), or are applicable to a limited number of 61 species. These findings are also complicated by the use of multiple methods by different studies 62 to evaluate and compare egg shape, with some more comprehensive in their evaluation of shape 63 differences than others (Havlíček et al., 2008).

65 Historically a range of methods have been applied to quantify egg shape; in morphometrics the 66 term 'form' refers to size plus shape, where 'shape' is defined as "all the geometric information

67 that remains when location, scale and rotational effects are filtered out from an object" (Kendall, 68 1977). Egg shape is often studied by computing ratios from linear distance measurements 69 (summarised by Preston, 1969; Narushin, 2001; Havlíček et al., 2008; Troscianko, 2014); the 
70 most common linear ratios used are egg elongation and asymmetry. Egg elongation describes the

71 divergence of an ellipse (flattening) from a circle, whereas asymmetry specifies the extent to

72 which one end of an egg is more pointed than the other. Egg elongation is calculated as the ratio

73 of egg length to breadth (maximum diameter) while egg asymmetry is calculated based on the

74 ratio between the distance from the lower vertex of the egg to the point where the polar axis

75 intersects the equatorial axis to egg length, as described by Deeming and Ruta (2014). Using

76 ratios, such as elongation and asymmetry, to describe egg shape are still widely used despite

77 their limitations. For example, two eggs with the same elongation index do not necessarily imply

78 the same shape, because the same ratio may be obtained if the widest point is at the middle of the

79 egg, or towards the base (Hoyt, 1979). Consequently, multiple indices have been used recently in

80 combination to obtain a better description of egg shape. However, these variables are likely to be

81 correlated with one another, leading to multiple difficulties in identifying the extent of shape

82 variation between samples and selecting which indices best characterise egg shape for a given

83 species (Narushin, 2001).

84

85 Variation in egg curvature is another important component of eggshell shape. Eggshell curvature

86 has been typically analysed based on a two-dimensional (2D) profile of the egg along its

87 longitudinal axis (pole to pole), and was superficially estimated in the past based on direct linear

88 measurements and mathematical equations to approximate the profile of an egg (Hutt, 1938;

89 Bonnet \& Mongin, 1965; Besch, Sluka \& Smith, 1968; Carter, 1968). However, this

90 methodology is often tedious to apply, and it is difficult to determine how closely the calculated

91 curvature mimics the true profile of the egg (Carter, 1970). Other methods have been recently

92 developed in an attempt to capture the precise geometry of such contours. That is the case of 
93 curve-fitting equations, which have been used to estimate egg shape through $2 \mathrm{D}$ contour profiles

94 (Nedomova, Severa \& Buchar, 2009; Troscianko, 2014), with some requiring separate equations

95 for each species (Preston, 1953, 1968; Smart, 1991) or were limited to specific shapes (Baker \&

96 Brawn, 2002). A comprehensive study on avian egg shape variation by Stoddard et al. (2017),

97 which incorporated 1400 bird species, was forced to emit irregular or extremely pointed or

98 asymmetric eggs owing to limitations in the curve-fitting equations used, thereby constraining

99 the apparent variation in egg shape.

100

101 Even though the use of 2D methods to capture egg shape remains widespread today and will

102 likely remain common for many years owing to its simplicity and low cost, our reliance on using

$1032 \mathrm{D}$ outlines to represent the complex three-dimensional (3D) geometry of eggs poses several

104 limitations. Firstly, 2D analyses rely on an assumption of radial symmetry of the eggshell, where

105 the plane of symmetry is considered to remain the same with rotation along the egg's

106 longitudinal axis (Deeming \& Ruta, 2014). However, assuming complete radial symmetry can be

107 misleading, as it often ignores subtle differences in curvature around the entire eggshell. Preston

108 (1969) acknowledged that irregularities in an egg such as creases, ridges or dents (Preston, 1968)

109 can reduce the accuracy of certain shape parameter measurements. Instances of extreme

110 deviation from radial symmetry, as documented in the common guillemot Uria aalgae

111 (Birkhead, 2016), have not allowed these irregular eggs to be included in 2D shape analysis.

112 Secondly, the 2D approach is prone to error associated with aligning a highly-rounded object

113 exactly parallel to the imaging plane (e.g., camera lens for photographs) (Troscianko, 2014), and

114 in many cases, no attempt is made to align the eggs (e.g., Stoddard et al., 2017). The centre of

115 gravity of most eggs is towards the pointed pole, causing this end of the egg to tilt downwards 
116 (for blown and fresh eggs) when placed on a horizontal surface in its resting position (Mao et al.,

117 2007). The potential error caused by egg misalignment for $2 \mathrm{D}$ analysis are expected to be

118 greatest for elongated or pointed eggs, as the outlines formed by these shapes are more

119 susceptible to small deviations in the angle of the egg's longitudinal axis relative to the imaging

120 plane. Careful egg alignment is needed to ensure $2 \mathrm{D}$ silhouettes provide an accurate

121 representation of true egg shape (Attard et al., 2017) but, undoubtedly, a 2D approach will still

122 entail certain amount of error when quantifying shape variables. Therefore, in order to fully

123 understand the function of various shapes and overcome the limitations listed above, we need a

124 robust and reliable method to quantify the complete naturally occurring range of egg shapes and

125 curvatures in 3D space.

126

127 When studying egg shape in a comparative context, it is important that within- and between-

128 clutch variation in egg characteristics are considered (Garamszegi \& Møller, 2010). Generally, it

129 is assumed that phenotypic variation among taxa is greater than within-species variation

130 (Felsenstein, 1985). However, phenotypic plasticity, population differences or measurement

131 errors are all known to contribute to within-species variability (Ives, Midford \& Garland, 2007),

132 which can lead to misinformed conclusions if sample sizes are low. A study of five species of

133 bird found that randomly selecting one egg from each clutch to measure length, breadth and

134 curvature around each pole was more representative of a species than sampling all eggs from

135 fewer clutches (Preston, 1968). Sampling bias can also occur in studies where all eggs are 136 sampled from different sized clutches. Such an experimental design would be biased in favour of 137 eggs collected from larger clutches (Preston, 1968). 
139 Egg shape variation is expected to be low within a species if shape strongly influences the

140 survival of the young (Clark, Ewert \& Nelson, 2001). Alternatively, if shape is relatively

141 unimportant for egg hatchability and subsequent offspring survival, then large variation in egg

142 shape within a given species might be expected. Egg shape can increase offspring survival by

143 improving egg strength (Gosler, Higham \& Reynolds, 2005), embryo growth (Deeming, 2017),

144 incubation efficiency (Drent, 1975; Rokitka \& Rahn, 1987; Deeming \& Ferguson, 1991; Barta \&

145 Székely, 1997; Mao et al., 2007; Šálek \& Zárybnická, 2015), and the detection of brood

146 parasitism (Underwood \& Sealy, 2006; Zölei et al., 2012; Attard et al., 2017). In contrast, within-

147 clutch variation in egg shape is predicted to be a reflection of parental investment in each

148 offspring (dependent on the female's breeding condition and experience) (Coulson, 1963), or

149 external factors influencing offspring survival (Briskie \& Sealy, 1990). Since spherical eggs have

150 the smallest surface area of all 3D solids of a given volume, reducing calcium investment in shell

151 production should therefore be advantageous to females that may be calcium limited (Gosler,

152 Higham \& Reynolds, 2005). Conversely, elongated eggs provide greater volume to facilitate

153 higher energy and nutrient storage capacity for embryo growth (Pearl \& Curtis, 1916), and thus,

154 provide the hatchling with greater fitness potential (Rose, Simpson \& Manning, 1996). Thus if

155 natural selection is acting on egg shape, we expect that differences in eggshell shape will reflect

156 adaptations to optimise incubation and/or hatchability (Hoyt, 1976).

158 In this study, micro computed tomography (micro-CT) data was used to generate 3D models, and

159 geometric morphometrics of 3D landmarks was used to quantify egg shape in four Australian

160 bird species (four clutches per sp.): the grey shrike-thrush Colluricincla harmonica, red-browed

161 finch Neochmia temporalis, spiny-cheeked honeyeater Acanthagenys rufogularis and superb 
162 fairy-wren Malurus cyaneus (Figure 1A). Our aim was to investigate whether there is greater

163 shape variation within a clutch than between clutches for a given species, and test if egg shape

164 can be used to distinguish between bird species. Using this dataset as a case study, we apply a 3D

165 landmark method to accurately quantify natural variation in egg shape. Our method builds upon

166 recent developments that attempt to precisely measure the $2 \mathrm{D}$ contours of the eggshells from

167 digital photographs using Elliptic Fourier analyses and semilandmark-based geometric

168 morphometric methods (Johnson, Leyhe \& Werner, 2001; Havlíček et al., 2008; Bravo \&

169 Marugán-Lobón, 2013; Murray et al., 2013; Deeming \& Ruta, 2014; Deeming, 2017). These

170 latest developments capture the egg outline and turn the contours into 2D shape variables (Iwata

171 \& Ukai, 2002; Murray et al., 2013) however they are often not as accurate as a 3D approach

172 (Loy et al., 2000; Sheets et al., 2006).

173

174 Method

175

$176 \underline{\text { Specimens }}$

177 Egg shape was compared for taxa from four distinct families: the Maluridae (fairy-wrens),

178 Meliphagidae (honeyeaters), Pachycephalidae (whistlers and thickheads) and the Estrildiae (wax

179 bills, grass finches, munias and allies) respectively (Table S1). All four species included, except

180 the grey shrike-thrush, are endemic to Australia. These taxa cover a range of body sizes (10-

181 68g), diets, clutch size (2-5 eggs per clutch), life histories (pair breeders versus cooperative

182 breeders), and evolutionary origins (Australian Old Endemics versus more recent Eurasian

183 colonists) (Table S1). Within each species, four clutches from different collection locations were

184 preferentially selected to ensure that different clutches were not laid by the same breeding female 
185 (Figure 1B). All clutches within a given species are from the same subspecies, with the exception

186 of the superb fairy-wren, which had one clutch from subspecies leggei (E14555), and the others

187 from cyanochlamys. All egg clutches were provided by the Australian National Wildlife

188 Collection, Canberra. Collection date, location and clutch size were available for all eggs

189 included in this study (Table 1). Only clutches collected after dichlorodiphenyltrichloroethane

190 (DDT) was banned in 1987 were included to minimise the risk of reported pesticide-induced

191 changes in avian eggshell characteristics (Fry 1995), although, given their dietary preferences,

192 none of these taxa are likely to have been affected by this process in any case. All eggs from

193 each clutch were included in this study, with the exception of one of the seven eggs from clutch

194 10376, which was too damaged to reliably reconstruct digitally.

195

196 Micro-CT Scanning

197 The intact eggshells $(\mathrm{N}=55)$ were scanned using a compact desktop micro-CT scanner (SkyScan 198 1174, Bruker micro-CT, Kontich, Belgium) at the Australian National Wildlife Collection, 199 Canberra. The following scanning parameters were used: $50 \mathrm{kV}$ source voltage, $80 \mu \mathrm{A}$ source 200 current, 33.28 to $33.45 \mu \mathrm{m}$ pixel size, $360^{\circ}$ rotational angle, $0.8^{\circ}$ rotational step, $2.3 \mathrm{~s}$ exposure 201 time, 40\% sharpening, 2 frame averaging, random movement and flat field correction turned on 202 and a $0.5 \mathrm{~mm}$ aluminium filter. The isotropic voxel size used for each scan was specimen 203 specific and was based on the resolution required for the length of the egg to fill $90 \%$ of the field 204 of view.

205

206 The acquired shadow projections (16-bit TIFF format) were further reconstructed into 2D slices 207 of the structure of each sample using the NRecon software interface (v.1.6.9, Brueker micro-CT, 
208 Kontich, Belgium). The reconstruction parameters used were: smoothing (5\%), ring artefact 209 correction $(20 \%)$, beam hardening correction (100\%) and setting of contrast limits between 0 and

210 1.4. A $1.5 \mathrm{ml}$ eppendorf tube containing MilliQ water was included in all scans. Micro-CT

211 Hounsfield units (HUs) were calibrated using known water CT density $(\mathrm{HU}=0)$ during 2D slice

212 reconstruction. The cross-section slices were stored in DICOM format and are available on the

213 Figshare repository (https://figshare.com/s/3af9f0cf5346e9b881f6).

215 3D-Volume Reconstruction

216 Digital segmentation and solid meshing of micro-CT tomographs was performed with the 217 medical imaging software Mimics (v. 16.0) and 3-matic (v. 8.0), using protocols adapted from 218 Attard et al. (2014). In brief, thresholding and segmentation of egg micro-CT data was conducted 219 in mimics to create a 3D object of the eggshell. The 3D object was imported in 3-matic where 220 the geometry was wrapped, reduced and smoothed, while preserving egg shape and size without 221 data loss. All eggs had been blown cleanly through a hole. To fill in the hole, the surface around 222 the hole of each egg was selected and filled using the freeform tool in 3-matic. The eggshell was 223 separated into two surfaces; one of the interior eggshell surface and one of the exterior eggshell 224 surface. A surface mesh of the exterior eggshell surface consisting of approximately 10,000 225 triangles was produced for each specimen and exported as a .PLY file for shape analysis, and are 226 available on Figshare (https://figshare.com/s/3af9f0cf5346e9b881f6).

Quantifying Egg Shape

229 We placed 206 landmarks on the 3D surface models to cover the contours of the egg using a 230 digitising routine written in R v.3.2.2 (Team, 2015) modified from those in the geomorph library 
231 (Adams \& Otárola-Castillo, 2013; Adams, Collyer \& Sherratt, 2016). Firstly, the egg model was

232 centred and rotated by its principal axes so that the x-axis lay along the longest dimension of the

233 egg. The top (pointed pole) and bottom (blunt pole) of the egg were defined by dividing the egg

234 into two halves and calculating the volumes (convex hull volume) of each; the top of the egg is

235 defined as the smaller half. Two landmarks were placed at the poles by taking the minimum and

236 maximum values along the $\mathrm{x}$-axis when $\mathrm{y}$ is zero. Four landmarks were placed on the $\mathrm{y}-$ and $\mathrm{z}$ -

237 axes by the same means, resulting in 6 landmarks that together demark the height, width and

238 depth, and are positionally homologous on every egg (Figure 2, red points). Then, 200 equally-

239 spaced semilandmarks were fitted to the first shell surface (Figure 2, black points) following the

240 algorithm outlined in Gunz et al. (2005) and Mitteroecker and Gunz (2009), producing a

241 template that could be fitted to every other specimen by thin-plate spline (TPS) warping

242 (Bookstein, 1989), using the 6 polar landmarks to orient the template. This method assures that

243 positional homology is maintained between every semilandmark and works well with curved 3D

244 structures with few homologous landmarks (Sherratt et al., 2014, 2016).

246 The landmark data were aligned using a generalised Procrustes superimposition (Rohlf \& Slice,

247 1990); all 206 semilandmarks were permitted to slide in either direction on two planes tangential

248 to the surface in order to minimise bending energy between specimens (Gunz, Mitteroecker \&

249 Bookstein, 2005). Variation among observations was visualised using a principal components

250 analysis (PCA) and plotting the first two axes in a biplot. The benefit of landmark coordinates is

251 they retain the geometric information and allow biological interpretation of the observed shape

252 variation. We summarised the main shape changes described by the first two PC axes using the

253 surface warp approach (e.g., Drake \& Klingenberg, 2010; Klingenberg, 2013; Sherratt et al., 
254 2014); an egg model derived from micro-CT reconstruction was warped to the mean shape using

255 the TPS method, and then this reference egg was warped to the shapes representing the minimum

256 and maximum values of PCs 1 and 2. For 3D objects this is one of the most intuitive and visually

257 accessible ways to view the measured shape variation.

258

259 To compare the performance of the 3D landmark data to the two commonly used egg shape 260 parameters we calculated elongation and asymmetry from the landmark coordinates. Egg length

261 was calculated as the interlandmark distance between the pole landmarks, and egg width is the

262 interlandmark distance between the two landmarks on the y-axis, and elongation is the ratio of

263 these two (length/width). For asymmetry, we used a trigonometric approach to calculate the

264 distance from the lower vertex of the egg (landmark 1) to the point where the polar axis

265 intersects the equatorial axis (where the hypotenuse was the average of the interlandmark

266 distances between the two landmarks on the y-axis and the lower vertex).

267

268 To test for statistical differences in egg shape between species and between clutches of each

269 species, we used the Procrustes ANOVA evaluated for significance with the F-test (Goodall,

270 1991). This distance-based ANOVA (D-ANOVA) uses Procrustes distances among specimens

271 rather than explained covariance matrices among variables, but is statistically equivalent to a

272 regular analysis of variance and is beneficial for high-dimensional datasets since only the

273 number of individuals is important in the model. We evaluated a nested model of egg shape

274 species / clutches. Significance testing was achieved through permutation using a residual

275 randomisation permutation procedure involving 1000 permutations (Collyer, Sekora \& Adams, 276 2015). 
278 To quantify the amount of egg shape variation within-species, as well as within-clutches of each

279 species, we measured the dispersion of all observations around the mean shape for the group. For

280 shape data, this is the Procrustes Variance, which is the mean squared Procrustes distance of 281 each specimen from the average shape, and can be calculated as the sum of the diagonal 282 elements of the covariance matrix of that group (Zelditch, Swiderski \& Sheets, 2012). To test for 283 statistical differences in disparity between clutches, we calculated absolute differences in 284 Procrustes variances between clutches and used these as test statistics in a permutation 285 procedure, where the Procrustes variances residuals are randomised among groups. For each 286 species, 1000 permutations were performed.

287

288 All analyses were performed in R v.3.2.2 (Team, 2015) using the geomorph library v.3.0 (Adams 289 \& Otárola-Castillo, 2013; Adams, Collyer \& Sherratt, 2016). Digitising routines are provided in 290 the Figshare Repository (https:/figshare.com/s/45fee8f96179b19d7988).

\section{Results}

294 Our data indicate a broad range of egg shapes for all species studied (Figure 3A). The first two 295 PCs accounted for $83.8 \%$ of the total egg shape variation across species, and therefore a biplot of 296 the two axes provides a reasonable approximation of the egg morphospace for this study. The 297 remaining PCs each contributed less than 5\% of the total variation and are not discussed further. $298 \mathrm{PC} 1(73.3 \%)$ is associated with elongation of the egg; negative PC1 scores correspond with 299 shorter, squatter eggs, while positive PC1 scores correspond with taller, more slender eggs. Eggs 
300 from all species were spread along the PC1 axis, revealing high variability in egg elongation

301 within each species. PC2 (10.5\%) is associated with tapering in egg shape, so called egg

302 asymmetry, with negative PC2 scores associated with eggs that were more asymmetrical and

303 positive PC2 scores associated with eggs that were more ovoid, with maximum egg breadth only

304 increasing slightly from low to high values of this axis. The two main axes therefore capture the

305 same general features that are commonly studied with 2D approaches. The correlation between

306 PC1 scores and the parameter of elongation is strong $(\mathrm{r}=0.99)$, and the correlation between PC2

307 scores and the parameter of asymmetry is moderate $(\mathrm{r}=0.66)$.

308

309 The four species had significantly different egg shapes based on 1000 iterations (Procrustes

310 ANOVA, $\mathrm{F}_{3,51}=4.248, \mathrm{P}<0.001$ ). For a nested design, there was no significant difference in

311 shape between species when clutches were considered, however clutches within-species are

312 significantly different (Table 2). Figure 3A shows clearly that there is substantial within-species

313 variation in egg shape for all species: within-species disparity (Procrustes variance) is

314 particularly high for the grey shrike thrush, red-browed finch and spiny-cheeked honeyeater

315 (Figure 4A). The high disparity in egg shape observed within-species is due to between-clutch

316 shape differences as well as within-clutch disparity (Figure 4B).

318 Clutch disparity appears quite variable within each species (Figure 4A), however the Procrustes

319 variances of clutches in all but three pairwise comparisons were not significantly different from

320 each other within each species (Figure 4B, Table 3). Overall mean clutch disparity was not

321 significantly different among species (ANOVA, $\left.\mathrm{F}_{3,12}=0.184, P=0.906\right)$. Together these results 
322 indicate that within-species egg shape variation is due to between-clutch differences as well as

323 within-clutch differences in egg shape.

324

325 Discussion

326

327 The variety of egg shapes extrapolated in this study concurred with those previously described

328 for each species in the literature. The only shape described for superb fairy-wren eggs is an

329 elongated oval, with markings commonly concentrated at the broader end (North, 1889;

330 Schodde, 1982; Campbell, 1990), implying that their eggs are only slightly rounder at one end.

331 In contrast, anecdotal accounts refer to several different shapes to describe eggs from the other

332 species included in this study (Marchant \& Higgins, 1990). The eggs of all grey shrike-thrush

333 subspecies have been described as either oval, rounded oval, stout oval to thick oval, or elongate-

334 oval, with some slightly pointed at each end (Marchant \& Higgins, 1990). Visualisation of the

335 egg shapes through PCA show that all grey shrike-thrush eggs incorporated in the present study

336 were at least slightly pointed at one pole, with some clutches clearly more elongated than others.

337 Eggs of the red-browed finch encompass a wide range of eggshell shapes (Figure 3), with this

338 species producing the largest clutch size of the species included in this study (Table 1). Egg

339 shapes of the Pied flycatcher Ficedula hypoleuca show high variability within populations,

340 ranging from pointed to ellipsoidal to nearly spherical, and is proposed to be linked to clutch size

341 (Kern \& Cowie, 1996).

342

343 Spiny-cheeked honeyeaters are reported to have oval or elongate-oval eggs (North, 1889;

344 Campbell, 1990), with spots and blotches usually concentrated at the broader end (North, 1889; 
345 Serventy \& Whittell, 1962; Campbell, 1990). Only one clutch of eggs from this species (E06324)

346 was very elongated, with all other eggs being relatively squat. This may be attributed to

347 geographic location; E06324 was from New South Wales, while the other clutches used from

348 this species were from South Australia (Figure 1B). Similarly, the grey shrike-thrush clutch

349 collected from South Australia (E14518) grouped together at the low end of PC1 values (Figure

3503 , red square symbol), signifying less elongated eggs compared to the three clutches collected

351 from New South Wales for this species (Figure 1B). It is possible that difference in climatic

352 conditions between geographic locations may be driving differences in egg elongation in these

353 species. In a study involving 310 native Australian passerines, avian eggs were found to be less

354 elongated in areas that were hot and dry or contained sparse plant canopies (Englert Duursma et

355 al., 2018). It was proposed that rounder eggs may be better adapted to hot and dry climatic

356 conditions by facilitating gas exchange between the embryo and the environment. This is

357 because spherical eggs have a lower surface area to volume ratio than ovoid eggs, and thus, may

358 potentially gain and lose heat more slowly, and lose less water. Whether geographical range may

359 be driving variation in egg shape within a species would benefit from additional work to test

360 variability in total shell porosity within a species for different egg shapes, and the influence of

361 egg shape (considering both elongation and asymmetry) on egg surface area to volume ratio.

362

363 Surprisingly, a large range of egg shapes was found within each species, resulting from

364 differences among clutches. Whether variation in egg shape between clutches for these species is

365 due to differences inherent in individual breeding females, or the location, climatic condition and

366 year of collection remains unknown. Further information and quantification of impacts of hatch

367 order and geographic location are required to increase our understanding of the processes 
368 involved in producing particular shaped eggs. We believe that the 3D methodological approach

369 demonstrated here has the potential to achieve this, allowing the quantification of fine-scale

370 differences in egg shape that may not be discernible using 2D methods. Our method can be

371 applied to any spherical to conical shaped object, including eggs from various taxa; from birds,

372 and extant reptiles to non-avian dinosaurs and pterosaurs.

373

374 The geometric morphometric approach demonstrated here is complementary to standard 2D

375 approaches (Deeming \& Ruta, 2014; Stoddard et al., 2017). We found that the main PC axes

376 summarising the highly multivariate dataset of landmark coordinates, which is a standard tool in

377 geometric morphometrics, discern the primary morphological traits measured in egg-shape

378 research: elongation and asymmetry. Our results indicate that elongation is captured in a similar

379 way by both methods, however asymmetry is less so (Figure 3). Given the 3D nature of an egg,

380 the way that the equatorial axis is defined in $2 \mathrm{D}$ will have important consequences on the

381 estimate of asymmetry. Our method mathematically aligns the 3D egg models by the principal

382 axes prior to digitisation, finding the maximum length, width and breadth of the egg. As such,

383 the landmarks in 3D capture different aspects of egg asymmetry than the traditional asymmetry

384 parameter does. Our study aligns with others that have shown that 3D landmark methods used on

385 highly-3D structures are preferable over 2D projections (e.g., Álvarez \& Perez, 2013; Cardini,

386 2014; Buser, Sidlauskas \& Summers, 2018).

388 Our method may also be of relevance to poultry science, enabling the inference of egg quality

389 based on egg geometry parameters (Narushin \& Romanov, 2002). The physical structure and

390 chemical composition of an eggshell is commonly used as an indicator of egg quality and 
391 stability during storage, with significant and direct effects on prices when eggs are graded

392 (Narushin \& Romanov, 2002). As egg quality traits are associated with hatchability and are

393 moderately heritable, it is important that birds producing eggs with desirable traits are retained

394 for future breeding (Ragozina, 1961; Rose, Simpson \& Manning, 1996). Physical factors

395 generally appreciated in eggs are shell appearance and strength, egg size, weight and the size and

396 appearance of the albumen and yolk (Murray et al., 2013). Such information can also be used to

397 provide tangible advice to farmers on the appropriate requirements for good quality eggs.

398 Previous studies have used the less accurate traditional linear measurements to evaluate egg

399 geometry parameters to infer egg quality of domesticated chickens. We believe that volumetric

400 methods such as micro-CT may provide a viable option to provide precise measurements of egg

401 volume and size, and eggshell thickness and shape. Egg quality traits are greatly influenced by

402 breeding and environmental factors (Clark, Ewert \& Nelson, 2001; Johnson, Leyhe \& Werner,

403 2001). As eggs are a primary source of animal protein globally, with levels of egg consumption

404 rising particularly in developing countries (Kern \& Cowie, 1996), it is important that new

405 scientific approaches are adopted to evaluate egg quality and production.

406

407 In biology, palaeontology and medicine, the demand for accurate 3D models of specimens is

408 continuously growing, as is the need to quantify and compare their geometry. Analysis of

409 accurate 3D models using a combination of techniques (e.g., geometric morphometrics and finite

410 element analysis) has enabled researchers to explore the function and evolution of anatomical

411 structures based on morphological differences in their shape (Polly et al., 2016). Both volume

412 and surface data are suitable for 3D geometric morphometrics and finite element analysis.

413 Volume and surface scanning are both non-contact methods, and therefore respond to increasing 
414 requirements in conservation, cultural heritage or repatriation programs. The absence of ionizing

415 radiation makes surface scanning a non-destructive/non-invasive measurement tool. Volume

416 scanning (CT, synchrotron, MRI, Terahertz, infrared) captures both the external and internal

417 structure of an object, whereas surface scanning is limited to the outer shell of an object. Surface

418 scanning refers to optical systems that measure objects through visible light (i.e., non-ionising

419 radiation). Photogrammetry and laser scanning are the two main sources that can provide surface

420 data. Low-cost photogrammetric methods are now available for high resolution topographic

421 reconstruction, ideally suited for low-budget research (Westoby et al., 2012). Any camera can be

422 used for photogrammetric purposes, depending on the accuracy requirements of the study

423 (Boehler \& Marbs, 2004). Using a set of images containing a high degree of overlap from a wide

424 array of positions, we have been able to capture the full three-dimensional structure of an egg

425 (see Figure S1). The camera positions and orientation of these images can be solved

426 automatically using image processing packages (e.g., Agisoft Photoscan, Memento,

427 CapturingReality, ImageModeler, Recap and 123D Catch) and a network of targets which known

428 3D positions can be assigned to scale the object to its real size (Westoby et al., 2012). A big

429 advantage of such packages is their simplified and user friendly interface, aimed mainly for non-

430 photogrammetrists. Photogrammetry provides an economical and efficient alternative to more

431 expensive volumetric imaging techniques such as CT or MRI, especially for capturing simple

432 objects at close range such as eggs. Laser scanners are usually preferred when digitising surfaces

433 of very complex, irregular objects. Laser scanner manufacturers normally provide their own

434 software, though an additional software package may also need to be purchased (Boehler \&

435 Marbs, 2004). The range of affordable methods now available to capture and analyse 3D data

436 will help facilitate future research on the form and function of biological structures. 


\section{Conclusions}

439

440 This study provides the first 3D analysis of egg shape and demonstrates its performance in

441 capturing shape variation at multiple biological levels. Our method uses a semi-automated

442 approach to digitise the entire surface of the eggshell to measure variation in shape between

443 specimens. This approach can be applied to any shaped egg as well as any spherical-to-conical

444 shaped object. The code for implementing this egg analysis technique is written using the $\mathrm{R}$

445 language and the script can be downloaded freely (see Data accessibility). Utilising a 3D-GM

446 approach offers an advantage over 2D approaches by improving the accuracy of shape, volume

447 and size measurements. Further research is required to evaluate the degree of error for different

$4483 \mathrm{D}$ and 2D approaches to measure egg shape.

449

450

Data accessibility

451

452 Micro-CT scans and surface meshes of all specimens included in this study (in DICOM and PLY

453 format, respectively) and the R scripts to perform the analyses are available on Figshare (see

454 https://figshare.com/s/3af9f0cf5346e9b881f6 and

455 https://figshare.com/s/45fee8f96179b19d7988).

456

457 Acknowledgements

458 
459 We thank Leo Joseph, Alex Drew and Margaret Cawsey from the Australian National Wildlife

460 Collection, Canberra for providing access to specimens and use of the micro-CT scanner. We

461 thank Dr Heinrich Mallison at the Museum für Naturkunde, Berlin, for sharing his expertise in

462 photogrammetry and Professor Tim Birkhead for providing access to specimens at the Alfred

463 Denny Museum, Sheffield.

464

465 References

466

467

468

469

470

471

472

473

474

475

476

477

478

479

480

Adams DC., Collyer ML., Sherratt E. 2016. geomorph: Software for geometric morphometric analyses. R package version 2.1.6. Available from URL http://cran.rproject.org/web/packages/geomorph/index.html.

Adams DC., Otárola-Castillo E. 2013. geomorph: an R package for the collection and analysis of geometric morphometric shape data. Methods in Ecology and Evolution 4:393-399. DOI: 10.1111/2041-210X.12035.

Álvarez A., Perez S. 2013. No Title. Evolutionary Biology 40:150-157.

Andersson M. 1978. Optimal egg shape in waders. Ornis Fennica 55:105-109.

Attard MRG., Medina I., Langmore NE., Sherratt E. 2017. Egg shape mimicry in parasitic cuckoos. Journal of Evolutionary Biology 30:2079-2084. DOI: 10.1111/jeb.13176.

Attard MRG., Parr WCH., Wilson LAB., Archer M., Hand SJ., Rogers TL., Wroe S. 2014. Virtual reconstruction and prey size preference in the mid cenozoic thylacinid, Nimbacinus dicksoni (Thylacinidae, Marsupialia). PLoS ONE 9. DOI: 10.1371/journal.pone.0093088.

Bain MM., Solomon SE. 1991. Cracking the secret of eggshells. New Scientist 129:27-29. 
481 Baker DE., Brawn J. 2002. A geometric method for determining shape of bird eggs. The Auk 482 119:1179-1186. DOI: 10.1642/0004-8038(2002)119[1179:AGMFDS]2.0.CO;2.

483 Barta Z., Székely T. 1997. The optimal shape of avian eggs. Functional Ecology 11:656-662. 484 DOI: $10.1046 /$ j.1365-2435.1997.00136.x.

485 Besch EL., Sluka SJ., Smith AH. 1968. Determination of surface area using profile recordings. 486 Poultry Science 47:82-85. DOI: 10.3382/ps.0470082.

487 Birkhead T. 2016. The Most Perfect Thing: Inside (and Outside) a Bird's Egg. Bloomsbury $488 \quad$ Publishing.

489

490

Birkhead TR., Thompson JE., Jackson D., Biggins JD. 2017. The point of a Guillemot's egg. Ibis 159:255-265.

Boehler W., Marbs A. 2004. 3D scanning and photogrammetry for heritage recording: a comparison. In: 12th International Conference on Geoinformatives - Geospacial Information Research: Bridging the Pacific and Atlantic. 291-298.

Bonnet Y., Mongin P. 1965. Mesure de la surface de l'œuf. Annales de zootechnie 14:311-317.

Bookstein FL. 1989. Principal warps: Thin-plate splines and the decomposition of deformations. IEEE Transactions on Pattern Analysis \& Machine Intelligence 11:567-585.

Bravo AM., Marugán-Lobón J. 2013. Morphometric analysis of dinosaur eggshells: constraints of size on shape. Historical Biology 25:697-704. DOI: 10.1080/08912963.2012.744989.

Briskie J V., Sealy SG. 1990. Variation in size and shape of Least Flycatcher eggs (Variaciones en el tamaño y forma de huevos de Empidonax minimus). Journal of Field Ornithology 61:180-191. 
502 Buser T., Sidlauskas B., Summers A. 2018. 2D or Not 2D? Testing the Utility of 2D Vs. 3D

503 Landmark Data in Geometric Morphometrics of the Sculpin Subfamily Oligocottinae

504 (Pisces; Cottoidea). The Anatomical Record 301:806-818. DOI: 10.1002/ar.23752.

505 Campbell AJ. 1990. Nest and eggs of Australian birds. Sheffield: Privately.

506 Cardini A. 2014. Missing the third dimension in geometric morphometrics: how to assess if 2D

507 images really are a good proxy for 3D structures? Hystrix, the Italian Journal of

508 Mammalogy 25:73-81. DOI: https://doi.org/10.1007/s11692-012-9194-3.

509 Carter TC. 1968. The hen's egg: A mathematical model with three parameters. British Poultry

$510 \quad$ Science 9:165-171. DOI: 10.1080/00071666808415706.

511 Carter TC. 1970. The hen's egg: Factors affecting the shearing strength of shell material. British

$512 \quad$ Poultry Science 11:433-449. DOI: 10.1080/00071667008415839.

513 Clark PJ., Ewert MA., Nelson CE. 2001. Physical apertures as constraints on egg size and shape

514 in the Common Musk Turtle, Sternotherus odoratus. Functional Ecology 15:70-77. DOI:

$515 \quad$ 10.1046/j.1365-2435.2001.00494.x.

516 Collyer ML., Sekora DJ., Adams DC. 2015. A method for analysis of phenotypic change for

517 phenotypes described by high-dimensional data. Heredity (Edinb) 115:357-365. DOI:

$518 \quad$ 10.1038/hdy.2014.75.

519 Coulson JC. 1963. Egg size and shape in the Kittiwake (Rissa tridactyla) and their use in

520 estimating age composition of populations. Proceedings of the Zoological Society of

521 London 140:211-226. DOI: 10.1111/j.1469-7998.1963.tb01861.x.

522 Deeming DC. 2017. Effect of composition on the shape of bird eggs. Journal of Avian Biology

523 48:1-7. DOI: $10.1111 /$ jav.01528. 
524 Deeming DC., Ferguson MWJ. 1991. Egg incubation: its effects on embryonic development in 525 birds and reptiles. Cambridge: Cambridge University Press.

526 Deeming DC., Ruta M. 2014. Egg shape changes at the theropod-bird transition, and a 527 morphometric study of amniote eggs. Royal Society Open Science 1:140311. DOI:

528

530

Drake AG., Klingenberg CP. 2010. Large-scale diversification of skull shape in domestic dogs: 10.1098/rsos.140311.

531

532

533

534

535

536

537

538

539

540

541

542

543

544

545 disparity and modularity. The American Naturalist 175:289-301. DOI: 10.1086/650372.

Drent RH. 1975. Incubation. In: Farner DS, King JR, Parkes KC eds. Avian biology. New York: Academic Press, 333-420.

Englert Duursma D., Gallagher R V., Price JJ., Griffith SC. 2018. Variation in avian egg shape and nest structure is explained by climatic conditions. Scientific Reports 8. DOI: 10.1038/s41598-018-22436-0.

Felsenstein J. 1985. Phylogenies and the comparative method. The American Naturalist 125:115.

Garamszegi LZ., Møller AP. 2010. Effects of sample size and intraspecific variation in phylogenetic comparative studies: a meta-analytic review. Biological Reviews 85:797-805. DOI: 10.1111/j.1469-185X.2010.00126.x.

Goodall C. 1991. Procrustes methods in the statistical analysis of shape. Journal of the Royal Statistical Society. Series B (Methodological) 53:285-339.

Gosler AG., Higham JP., Reynolds SJ. 2005. Why are birds' eggs speckled? Ecology Letters 8:1105-1113. DOI: 10.1111/j.1461-0248.2005.00816.x.

Gunz P., Mitteroecker P., Bookstein FL. 2005. Semilandmarks in Three Dimensions. In: Slice 
DE ed. Modern Morphometrics in Physical Anthropology. New York: Springer US, 73-98. DOI: 10.1007/0-387-27614-9_3.

548

549

550

551

552

553

554

555

556

557

558

559

560

561

562

563

564

565

566

567

Havlíček M., Nedomová Š., Simeonovová J., Severa L., Křivánek I. 2008. On the evaluation of chicken egg shape variability. Acta Universitatis Agriculturae et Silviculturae Mendelianae Brunensis 56:69-74. DOI: 10.11118/actaun200856050069.

Hoyt DF. 1976. The effect of shape on the surface-volume relationships of birds' eggs. Condor 78:343-349. DOI: $10.2307 / 1367694$.

Hoyt DF. 1979. Practical methods of estimating volume and fresh weight of bird eggs. The Auk $96: 73-77$.

Hutt FB. 1938. Embryonic mortality in the fowl: VII. On the relation of malpositions to the size and shape of eggs. Poultry Science 17:345-352. DOI: 10.3382/ps.0170345.

Ives AR., Midford PE., Garland T. 2007. Within-species variation and measurement error in phylogenetic comparative methods. Systematic Biology 56:252-270. DOI: 10.1080/10635150701313830.

Iwata H., Ukai Y. 2002. SHAPE: a computer program package for quantitative evaluation of biological shapes based on elliptic Fourier descriptors. The Journal of heredity 93:384-385. DOI: $10.1093 /$ jhered/93.5.384.

Johnson LS., Leyhe JE., Werner C. 2001. The shape of eggs in different-sized clutches of the house wren (Troglodytes aedon). Canadian Journal of Zoology 79:1527-1531. DOI: 10.1139/z01-099.

Kendall DG. 1977. The diffusion of shape. Advances in Applied Probability 9:428-430. DOI: $10.2307 / 1426091$ 
568 Kern MD., Cowie RJ. 1996. The size and shape of eggs from a Welsh population of Pied

569 flycatchers: testing Hoyt's use of egg dimensions to ascertain egg volume (Tamaño y forma

570 de los huevos de una población de Ficedula hypoleuca: poniendo a pruebas la fórmula de

$571 \quad$ Hoyt para determina. Journal of Field Ornithology 67:72-81.

572 Klingenberg CP. 2013. Visualizations in geometric morphometrics: how to read and how to

573

574 make graphs showing shape changes. Hystrix, the Italian Journal of Mammalogy 24:15-24.

Loy A., Busilacchi S., Costa C., Ferlin L., Cataudella S. 2000. Comparing geometric morphometrics and outline fitting methods to monitor fish shape variability of Diplodus puntazzo (Teleostea: Sparidae). Aquacultural Engineering 21:271-283. DOI: 10.1016/S0144-8609(99)00035-7.

Mao KM., Murakami A., Iwasawa A., Yoshizaki N. 2007. The asymmetry of avian egg-shape: An adaptation for reproduction on dry land. Journal of Anatomy 210:741-748. DOI: 10.1111/j.1469-7580.2007.00737.x.

Marchant S., Higgins PJ. (eds.) 1990. Handbook of Australian, New Zealand and Antarctic Birds. Volume 1: Ratites to Ducks. Melbourne: Oxford University Press. 36:235-247. DOI: 10.1007/s11692-009-9055-x.

Murray CM., Piller KR., Merchant M., Cooper A., Easter ME. 2013. Salinity and egg shape variation: a geometric morphometric analysis. Journal of Herpetology 47:15-23. DOI: 10.1670/11-123.

Narushin VG. 2001. AP_-Animal Production Technology: shape geometry of the avian egg. 
591 Narushin VG., Romanov MN. 2002. Egg physical characteristics and hatchability. World's

592 Poultry Science 58:297-303. DOI: 10.1079/WPS20020023.

593 Nedomova S., Severa L., Buchar J. 2009. Influence of hen egg shape on eggshell compressive 594 strength. International Agrophysics 23:249-256.

595 North AJ. 1889. Nests and eggs of birds found breeding in Australia and Tasmania. Sydney:

596 Australian Museum.

597 Olsen PD., Cunningham RB., Donnelly CF. 1994. Avian egg morphometrics -allometric models 598 of egg volume, clutch volume and Shape. Australian Journal of Zoology 42:307-321. DOI: 599 10.1071/ZO9940307.

600

Pearl R., Curtis MR. 1916. Dwarf eggs of the domestic fowl. In: Maine agricultural experiment 601 station. Orono: University of Maine, 48.

602 Polly PD., Stayton CT., Dumont ER., Pierce SE., Rayfield EJ., Angielczyk KD. 2016.

603 Combining geometric morphometrics and finite element analysis with evolutionary 604 modeling: towards a synthesis. Journal of Vertebrate Paleontology 36:e1111225. DOI: $605 \quad 10.1080 / 02724634.2016 .1111225$.

606 Preston FW. 1953. The shapes of birds' eggs. The Auk 70:160-182.

607 Preston FW. 1968. The shapes of birds' eggs: mathematical aspects. The American 608 Ornithologists’ Union 85:454-463. DOI: 10.2307/4083294.

609 Preston FW. 1969. Shapes of Birds â€ $€^{\mathrm{TM}}$ Eggs : Extant North American Families. The Auk $610 \quad 86: 246-264$. DOI: $10.2307 / 4083498$. 
611 Ragozina MN. 1961. Development of hen's embryo in correlation with its yolk and egg

612 membranes. Moscow, Russia.

613 Rensch B. 1947. Neuere Probleme der Abstammungslehre. Enke.

614 Rohlf FJ., Slice D. 1990. Extensions of the procrustes method for the optimal superimposition of 615 landmarks. Systematic Zoology 39:40-59. DOI: 10.2307/2992207.

616 Rokitka MA., Rahn H. 1987. Regional differences in shell conductance and pore density of avian 617 eggs. Respiration physiology 68:371-376. DOI: 10.1016/S0034-5687(87)80021-X.

618 Rose FL., Simpson TR., Manning RW. 1996. Measured and predicted egg volume of Pseudemys 619 texana with comments on turtle egg shape. Journal of Herpetology 30:433-435. DOI: $620 \quad 10.2307 / 1565188$.

621 Šálek ME., Zárybnická M. 2015. Different temperature and cooling patterns at the blunt and 622 sharp egg poles reflect the arrangement of eggs in an avian clutch. PloS ONE 10:e0117728623 e0117728. DOI: 10.1371/journal.pone.0117728.

624 Schodde R. 1982. The Fairy-wrens. Melbourne: Lansdowue.

625 Serventy DL., Whittell HM. 1962. Birds of Western Australia. Perth: Paterson Brokensh Pty. 626 Ltd.

627 Sheets HD., Covino KM., Panasiewicz JM., Morris SR. 2006. Comparison of geometric 628 morphometric outline methods in the discrimination of age-related differences in feather 629 shape. Frontiers in zoology 3:15. DOI: 10.1186/1742-9994-3-15.

630 Sherratt E., Alejandrino A., Kraemer AC., Serb JM., Adams DC. 2016. Trends in the sand:

631 Directional evolution in the shell shape of recessing scallops (Bivalvia: Pectinidae).

632 Evolution; international journal of organic evolution 70:2061-2073. DOI: 
634 Sherratt E., Gower DJ., Klingenberg CP., Wilkinson M. 2014. Evolution of cranial shape in 635 caecilians (Amphibia: Gymnophiona). Evolutionary Biology 41:528-545. DOI:

$636 \quad 10.1007 / \mathrm{s} 11692-014-9287-2$.

637 Smart IHM. 1991. Egg-shape in birds. In: Deeming DC, Ferguson MWJ eds. Egg incubation: its 638 effects on embryonic development in birds and reptiles. Cambridge, New York: Cambridge 639 University Press, 101-116.

640 Stoddard MC., Yong EH., Akkaynak D., Sheard C., Tobias JA., Mahadevan L. 2017. Avian egg 641 shape: Form, function, and evolution. Science 356:1249-1254. DOI:

642 10.1126/science.aaj1945.

643

644 645 646 647 648

649

650

651

652

653

654

Team RC. 2015. R: a language and environment for statistical computing. Vienna, Austria: R Foundation for Statistical Computing. URL http://www.R-project.org/.

Troscianko J. 2014. A simple tool for calculating egg shape, volume and surface area from digital images. Ibis 156:874-878. DOI: 10.1111/ibi.12177.

Underwood TJ., Sealy SG. 2006. Influence of shape on egg discrimination in American robins and gray catbirds. Ethology 112:164-173. DOI: 10.1111/j.1439-0310.2006.01143.x.

Westoby MJ., Brasington J., Glasser NF., Hambrey MJ., Reynolds JM. 2012. “Structure-fromMotion" photogrammetry: A low-cost, effective tool for geoscience applications. Geomorphology 179:300-314. DOI: 10.1016/j.geomorph.2012.08.021.

Zelditch ML., Swiderski DL., Sheets HD. 2012. Geometric morphometrics for biologists: a primer. London: Academic Press.

Zölei A., Hauber ME., Geltsch N., Moskát C. 2012. Asymmetrical signal content of egg shape as 
655 predictor of egg rejection by great reed warblers, hosts of the common cuckoo. Behaviour

656 149:391-406. DOI: 10.1163/156853912X638445. 
Figure 1

Photograph of eggs and geographical range of clutches included in this study.

(A) Photographs of eggs from the four species of bird included in this study; from left to right, grey-shrike thrush, red-browed finch, spiny-cheeked honeyeater and superb fairy-wren. (B) Geographical range of bird clutches. See Table 1 for symbol shape used to represent each clutch. 


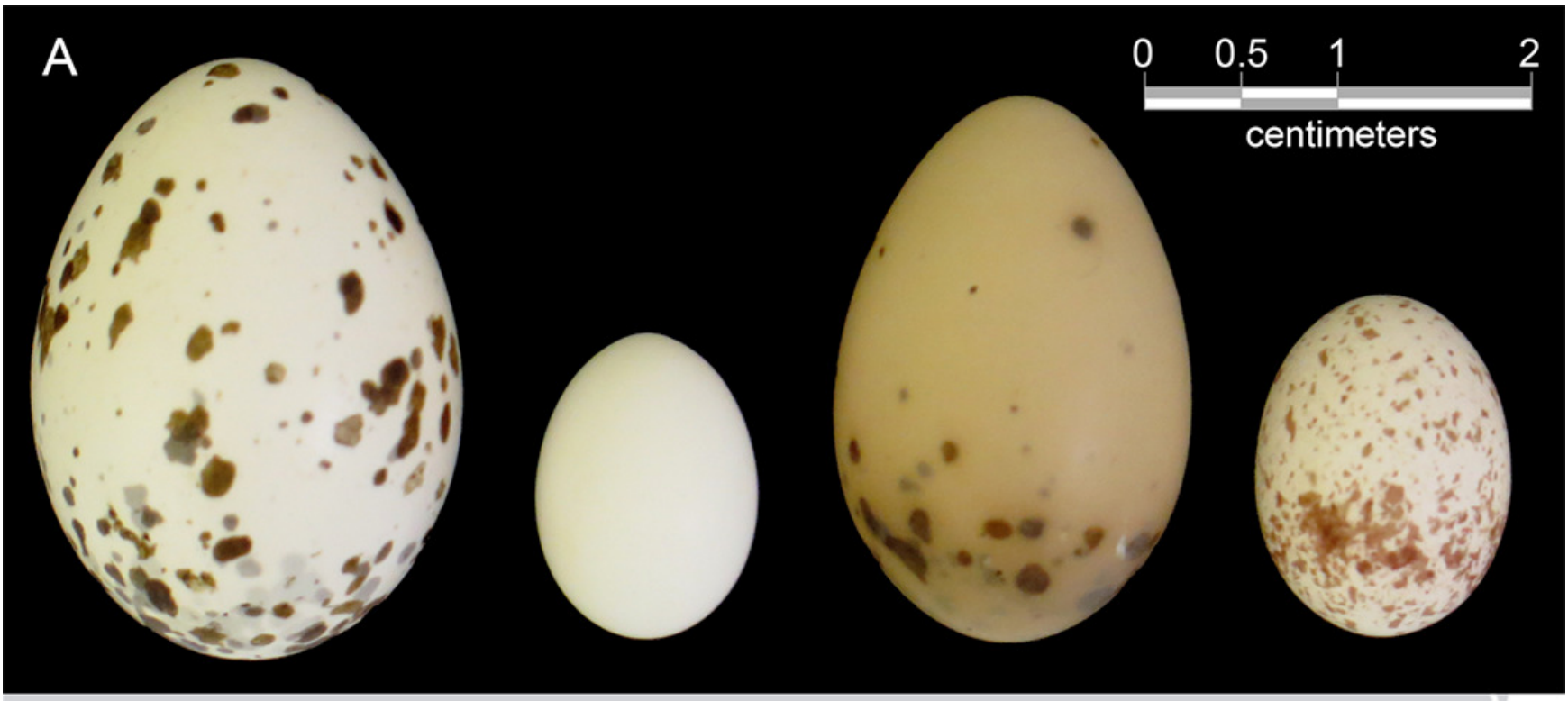

B

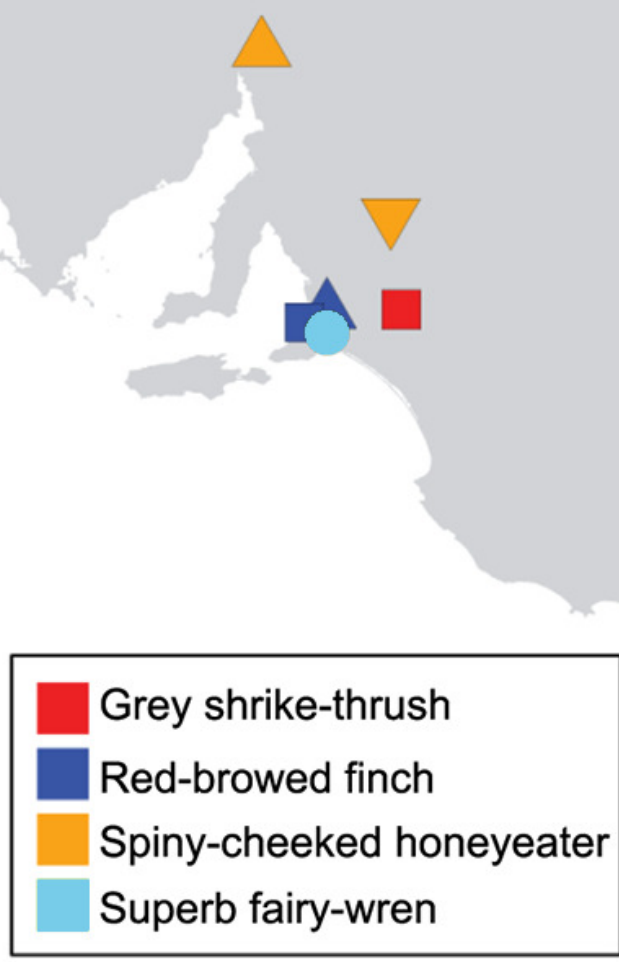

0

500

Peerj reviquing phetle(2015:09:6650:2:0:NEW 31 May 2018) 


\section{Figure 2 (on next page)}

Example of the digitisation of landmarks on a virtual egg.

The position of six homologous landmarks (pointed pole, blunt pole and four equidistant points around equator) are shown in red, and the template of semilandmarks are shown in black. The surface mesh of the egg was reconstructed from micro-CT data. 


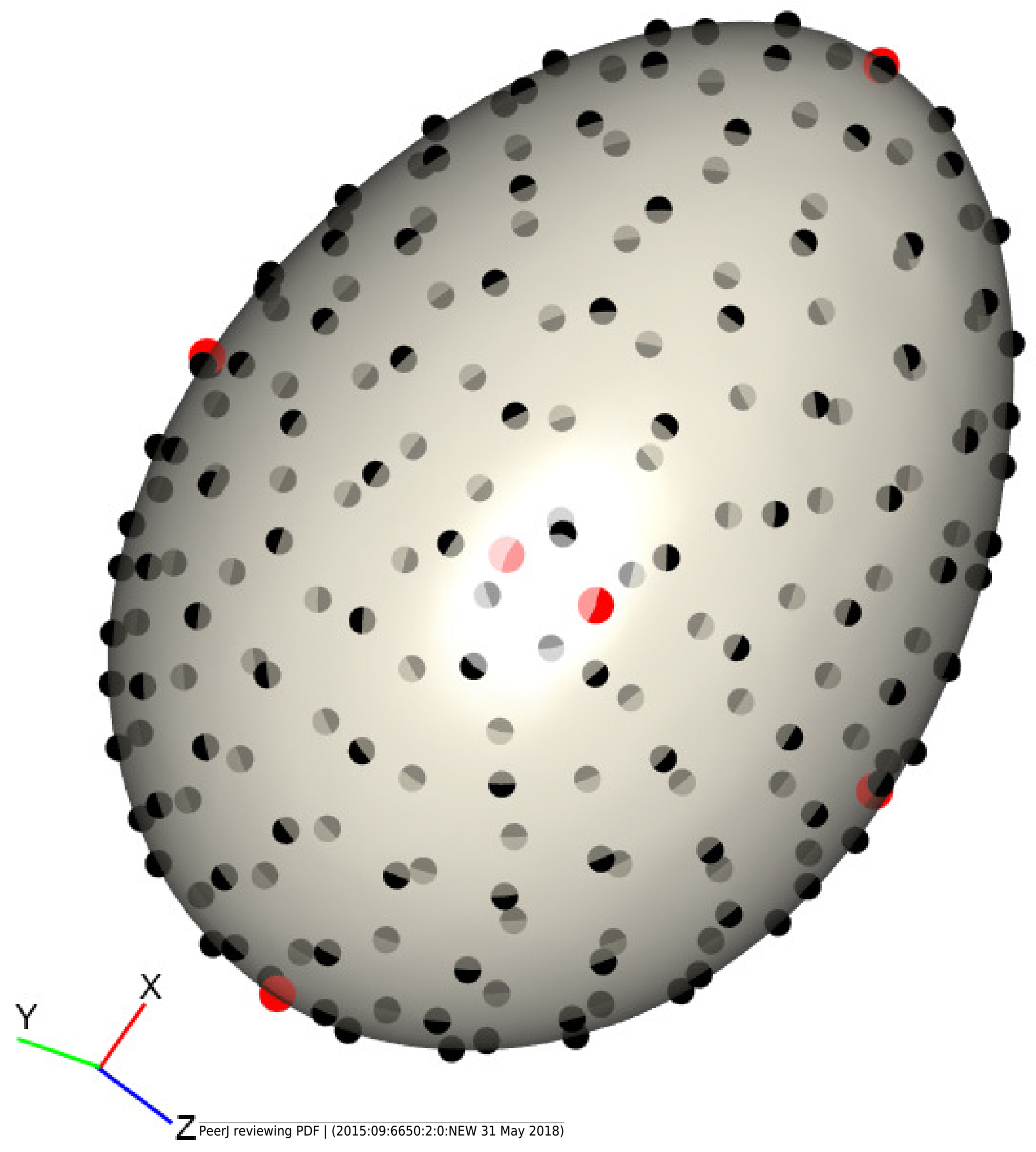




\section{Figure 3}

Comparison between 3D and 2D approach to quantify egg shape variation among four bird species.

Each symbol in shape space represents a single egg. Proximity of each symbol indicates similarity in shape. Symbol colour represents species and shape represents clutch (Table 1). (A) Morphospace defined by the two first principal components (PC's) of shape variance using 3D landmark data. The percentage of total variance described by each axis is shown in parentheses. Shapes associated with the extreme ends of each PC axis are shown as warped surface models (see text for details). The origin point corresponds to the mean shape. (B) Morphospace of egg shape in two dimensions (elongation ratio and asymmetry ratio) using 2D linear measurements. 


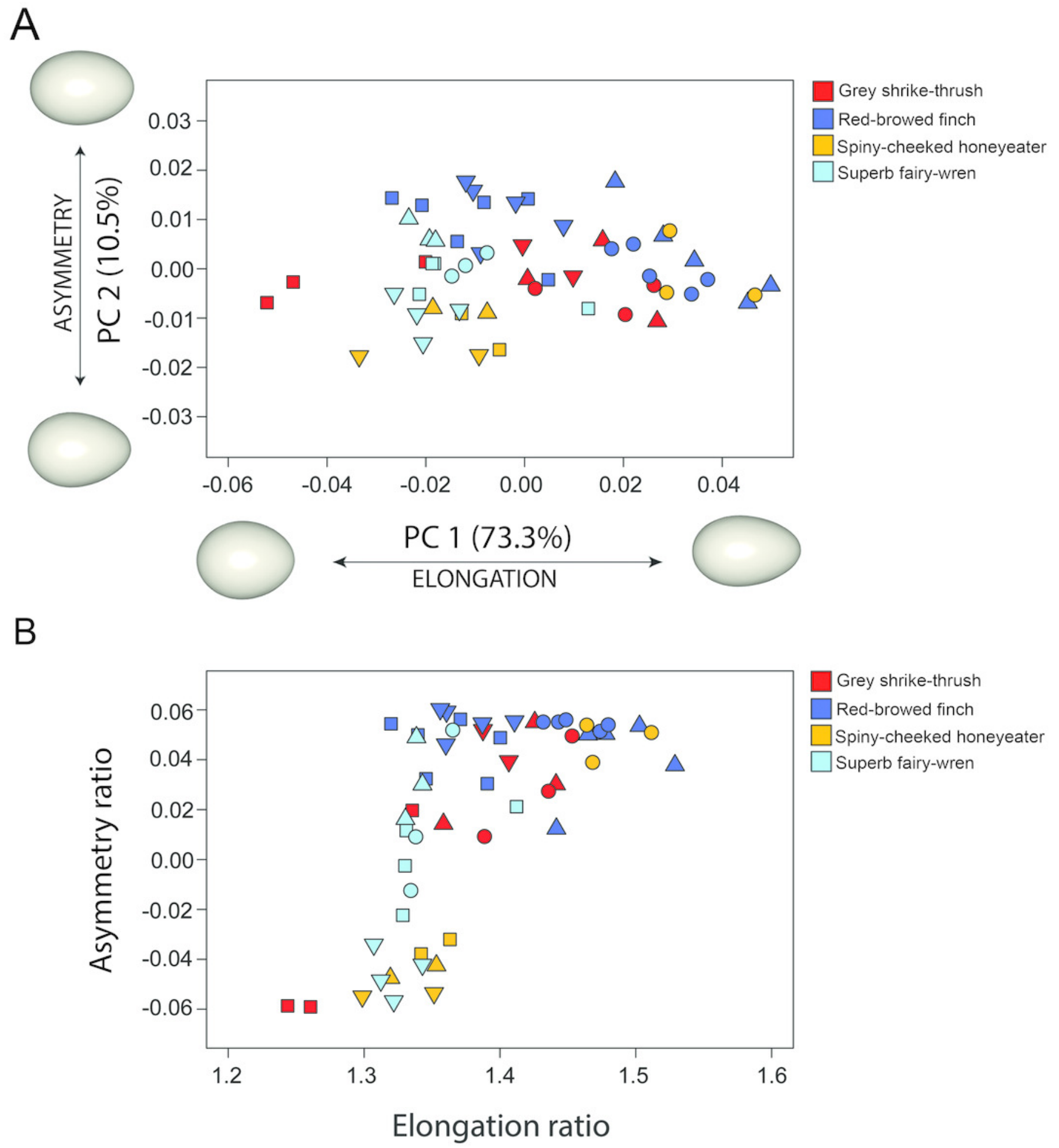




\section{Figure 4}

Bird egg shape disparity within species and clutches.

Disparity, measured as Procrustes variance, of egg shape within (A) species and (B) clutches. Colours correspond to species as in Figure 1. The $y$-axes of both graphs are plotted to the same scale. Pairwise comparisons of clutch disparity are presented in Table 4.

A

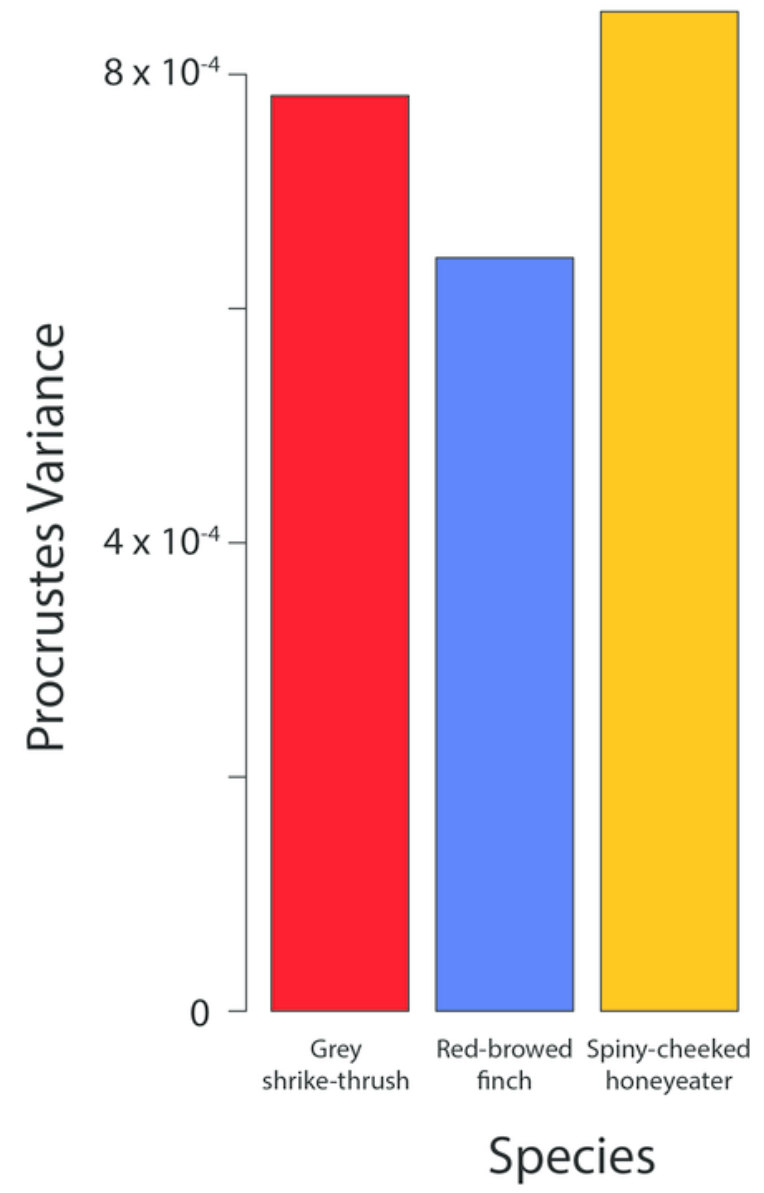

B

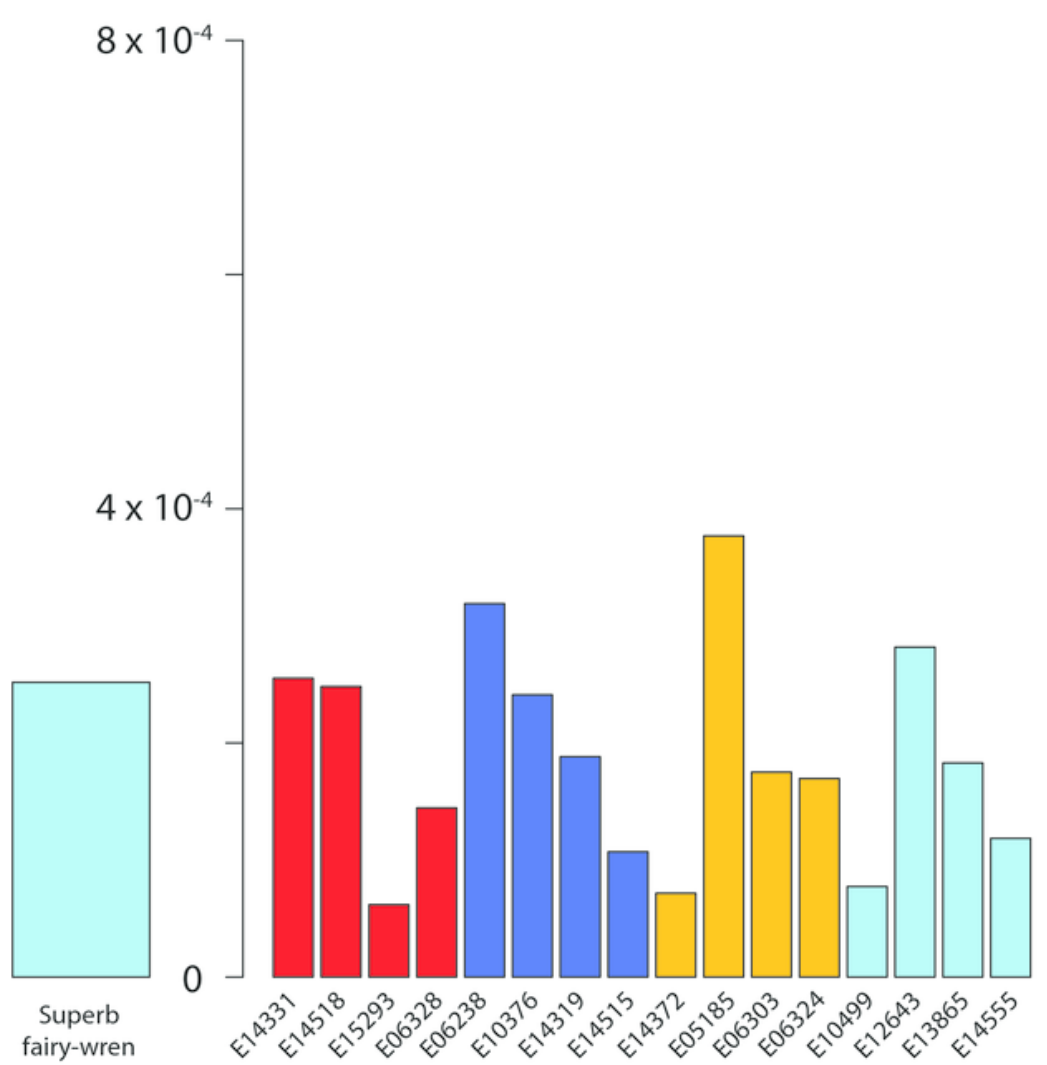

Clutch 


\section{Table $\mathbf{1}$ (on next page)}

Museum accession and collection information on each clutch included in the study. 


\begin{tabular}{|c|c|c|c|c|c|c|}
\hline Common name & $\begin{array}{l}\text { Catalogue } \\
\text { Number }\end{array}$ & $\begin{array}{l}\text { Clutch } \\
\text { size }\end{array}$ & Latitude & Longitude & $\begin{array}{l}\text { Date } \\
\text { collected }\end{array}$ & Symbol \\
\hline Grey shrike-thrush & E14331 & 3 & -34.96 & 149.17 & $14 / 01 / 2004$ & triangle \\
\hline Grey shrike-thrush & E14518 & 3 & -35.11 & 139.54 & 06/09/1991 & square \\
\hline Grey shrike-thrush & E15293 & 2 & -35.42 & 149.45 & $01 / 10 / 2006$ & inverted triangle \\
\hline Grey shrike-thrush & E06328 & 3 & -33.01 & 147.92 & $15 / 09 / 1999$ & circle \\
\hline Red-browed finch & E06238 & 5 & -35.1 & 138.73 & $27 / 10 / 1987$ & triangle \\
\hline Red-browed finch & E10376 & 6 & -35.28 & 138.57 & 08/11/1988 & square \\
\hline Red-browed finch & E14319 & 5 & -34.57 & 150.77 & $11 / 12 / 2003$ & inverted triangle \\
\hline Red-browed finch & E14515 & 5 & -35.22 & 149.13 & $03 / 01 / 2005$ & circle \\
\hline Spiny-cheeked & E14372 & 2 & -32.19 & 138.02 & $12 / 09 / 1988$ & triangle \\
\hline honeyeater & & & & & & \\
\hline $\begin{array}{l}\text { Spiny-cheeked } \\
\text { honeyeater }\end{array}$ & E05185 & 2 & -30.56 & 138.98 & $17 / 09 / 1987$ & square \\
\hline $\begin{array}{l}\text { Spiny-cheeked } \\
\text { honeyeater }\end{array}$ & E06303 & 2 & -34.1 & 139.43 & $27 / 08 / 1992$ & inverted triangle \\
\hline $\begin{array}{l}\text { Spiny-cheeked } \\
\text { honeyeater }\end{array}$ & E06324 & 3 & -32.97 & 146.15 & $14 / 09 / 1999$ & circle \\
\hline Superb fairy-wren & E10499 & 3 & -35.22 & 149.13 & $15 / 10 / 2002$ & triangle \\
\hline Superb fairy-wren & E12643 & 4 & -26.97 & 151.5 & $15 / 09 / 2002$ & square \\
\hline Superb fairy-wren & E13865 & 4 & -34.55 & 150.73 & $31 / 10 / 1998$ & inverted triangle \\
\hline Superb fairy-wren & E14555 & 3 & -35.34 & 138.69 & $16 / 11 / 1992$ & circle \\
\hline
\end{tabular}




\section{Table 2 (on next page)}

Nested D-ANOVA evaluating variation in shape between species and between clutches within each species.

P-values based on 1000 random residual permutations. 


$$
\begin{array}{lllllll}
\text { Df } & \text { SS } & \text { MS } & \mathrm{R}^{2} & \mathrm{~F} & \mathrm{Z} & \operatorname{Pr}(>\mathrm{F})
\end{array}
$$

\begin{tabular}{|c|c|c|c|c|c|c|c|}
\hline species & 3 & 0.008318 & 0.00277278 & 0.19978 & 1.4768 & 0.4613 & 0.324675 \\
\hline species:clutch & 12 & 0.022531 & 0.00187758 & 0.54112 & 6.7875 & 6.1576 & 0.000999 \\
\hline Residuals & 39 & 0.010788 & 0.00027662 & 0.25910 & & & \\
\hline Total & 54 & 0.041638 & & & & & \\
\hline
\end{tabular}




\section{Table 3(on next page)}

Pairwise comparisons of Procrustes variance between clutches.

Values in the lower triangle are the observed pairwise absolute differences (distances) among clutch Procrustes variances. Upper triangle values are P-values associated with pairwise differences (1000 permutations). P-values in bold are significant at the $5 \%$ level. 


\section{Grey shrike-thrush (Colluricincla harmonica)}

\begin{tabular}{ccccc} 
& E14331 & E14518 & E15293 & E06328 \\
\hline E14331 & - & 0.9570 & 0.0859 & 0.3237 \\
E14518 & $7.06 \mathrm{E}-06$ & - & 0.1039 & 0.3766 \\
E15293 & $1.93 \mathrm{E}-04$ & $1.86 \mathrm{E}-04$ & - & 0.5005 \\
E06328 & $1.11 \mathrm{E}-04$ & $1.04 \mathrm{E}-04$ & $8.27 \mathrm{E}-05$ & - \\
\hline
\end{tabular}

Red-browed finch (Neochmia temporalis)

\begin{tabular}{ccccc} 
& E06238 & E10376 & E14319 & E14515 \\
\hline E06238 & - & 0.4266 & 0.1678 & $\mathbf{0 . 0 2 5 0}$ \\
E10376 & $7.79 \mathrm{E}-05$ & - & 0.5794 & 0.1299 \\
E14319 & $1.31 \mathrm{E}-04$ & $5.28 \mathrm{E}-05$ & - & 0.3766 \\
E14515 & $2.12 \mathrm{E}-04$ & $1.34 \mathrm{E}-04$ & $8.13 \mathrm{E}-05$ & - \\
\hline
\end{tabular}

Spiny-cheeked honeyeater (Acanthagenys rufogularis)

\begin{tabular}{ccccc} 
& E14372 & E05185 & E06303 & E06324 \\
\hline E14372 & - & $\mathbf{2 . 5 0 E - 0 3}$ & 0.3861 & 0.3951 \\
E05185 & $3.05 \mathrm{E}-04$ & - & 0.0919 & $\mathbf{0 . 0 4 9 0}$ \\
E06303 & $1.03 \mathrm{E}-04$ & $2.02 \mathrm{E}-04$ & - & 0.9755 \\
E06324 & $9.80 \mathrm{E}-05$ & $2.07 \mathrm{E}-04$ & $5.47 \mathrm{E}-06$ & - \\
\hline
\end{tabular}

Superb fairy-wren (Malurus cyaneus)

\begin{tabular}{ccccc} 
& E10499 & E12643 & E13865 & E14555 \\
\hline E10499 & - & 0.0829 & 0.4206 & 0.6523 \\
E12643 & $2.04 \mathrm{E}-04$ & - & 0.4615 & 0.2138 \\
E13865 & $1.06 \mathrm{E}-04$ & $9.90 \mathrm{E}-05$ & - & 0.5495 \\
E14555 & $4.12 \mathrm{E}-05$ & $1.63 \mathrm{E}-04$ & $6.43 \mathrm{E}-05$ & - \\
\hline
\end{tabular}

